\title{
A Review of the Calculation Methods of Lifting Capacity in Wind Loads on Ocean Platforms
}

\author{
Yuchen Sheng \\ School of Ocean Science and Engineering, Shanghai Maritime University, Shanghai, China \\ Email: yuchen199602@163.com
}

How to cite this paper: Sheng, Y.C. (2021) A Review of the Calculation Methods of Lifting Capacity in Wind Loads on Ocean Platforms. Open Journal of Marine Science, 11, 69-79.

https://doi.org/10.4236/ojms.2021.112005

Received: February 5, 2021

Accepted: April 3, 2021

Published: April 6, 2021

Copyright (c) 2021 by author(s) and Scientific Research Publishing Inc. This work is licensed under the Creative Commons Attribution International License (CC BY 4.0).

http://creativecommons.org/licenses/by/4.0/

(c) (i) Open Access

\begin{abstract}
Wind load is a control load that affects the safety of structures in the design of ocean platforms. It has not only direct and powerful effects that may cause structure resonance but also has indirect effects causing waves or currents in the ocean. By analyzing the domestic and international norms, this study presents a review of calculation methods of wind load on ocean platforms, which belongs to large-scale non-entity structure used in the open sea while surrounding wind has no fixed direction. Current computations according to the norms are not accurate, which even not takes the force of the wind against the surface perpendicular to the structure into consideration. Additionally, this study also introduces and compares the lift model of platforms based on different theories, such as vortex-excitation and vibration, engineering structure dynamics, gas flow pressure theory, analyzing their applicability, advantages, and disadvantages. This paper analyzes the limitations and applicable conditions of the existing calculation method itself, such as the lift model is suitable for the existence of stable vortex wake; the calculation method of the structural dynamics of marine engineering must be combined with the wind tunnel test and consider the mistakes caused by the position relationship; the numerical simulation method is accurate but tedious. This study provides an insight into the calculation methods of lift in designing ocean platforms, including the finite element method for simulating fluid force and updating formulas in Chinese norms.
\end{abstract}

\section{Keywords}

Lift, Wind Load, Calculation Method, Lift Model, Structural Dynamics, Vortex-Excitation

\section{Introduction}

In recent decades, with the consumption of oil and gas resources inland, the 
global demand for oil and gas resources has been increasing. Existing research shows that the oceans account for 70 percent of the world's oil and gas reserves. Therefore, the ocean will become the main competitive direction of oil and gas resource exploitation. The infrastructure for the exploitation of offshore oil and gas resources includes large structures such as offshore coastal engineering and deep-sea marine platforms. Among these structures, with the exploitation and consumption of offshore resources, the development of deep-sea marine platforms will become an inevitable trend.

The ocean platform is a structure that provides production and living facilities for Drilling, oil production, transportation, observation, navigation, construction, or other activities at sea, mainly composed of the upper structure, facilities, and equipment, support structure, etc. It has complex construction and multiple components and is so expensive that it must maintain safety even in the extreme situation of the maximum winds and waves during its service period [1].

The service environment of the marine platform is harsh, and it must resist the load of the marine environment such as wind, waves and currents. Therefore, the design of ocean platforms must take full account of environmental loads. Among these environmental loads, the effect of wind load is the most obvious, but the existing design and calculation methods are not accurate, and it is urgent to elaborate and compare the relevant calculation methods of wind load.

The environmental conditions and behavior of deep-sea marine platforms are different from the land structure. On the one hand, the wind environment of the ocean is more severe and complex, its intensity is greater, the wind profile characteristics, pulsation wind characteristics, wind speed stability characteristics, and other wind field characteristics are also very different. On the other hand, the wind resistance effect of marine structures and land-based structures is different, and its bottom is in a metatable floating state, which needs to consider the combined effect of waves and currents.

Wind load is the main load of the upper structure and the control load of the whole structure, and it is also the most common reason for the overturning of mobile ocean platforms. It has not only stress effects that may cause structure resonance but also indirect effects causing waves or currents in the ocean [2]. The ocean platform belongs to a large-scale non-entity structure used in the open sea areas while the surrounding wind has no fixed direction. In conventional calculations, the lift effect is ignored, assuming that the wind-tilt torque is a cosine function at an averse angle and that only lateral wind-dumping loads are calculated. However, this assumption results in large deviation sometimes. In conclusion, conventional computing has been found with lots of problems, and the lift of the ocean platform under wind load must be taken into account.

At present, the "meteorological standards" [3] are used to measure the stability of domestic and foreign ocean platforms, which means to express environmental load to wind load and consider the impact of waves on the platform and other factors into the stability criterion. However, due to the structural characteristics of platforms, the wind load determination is much more complex than 
conventional hulls. Differing from drilling vessels, there are air gaps under the deck of platforms, which will have a significant lifting effect when affected by the wind [4]. When the platform is tilted, the bottom of the decks will also be counted to the wind-affected area [5]. To find out the most dangerous tip-over direction of the platform, wind loads in different overturning directions must be calculated.

\section{Status of Research}

The upper tower of the marine platform has the characteristics of high height, soft structure, light mass, and low self-vibration frequency, and the wind load is the main load of its structural carrying capacity. Research methods include theoretical analysis, wind tunnel test, field measurement, and numerical simulation.

In the past 70 years, wind theory was created and developed, which has been widely used in wind resistance research of engineering structures. The existing wind load calculation methods include the equivalent calculation method used in the norms, coefficient value method based on reliability theory, dynamic response method, hydrodynamics theory calculation method, etc.

Besides, the field measurement method is the most direct means to study the wind resistance of structures, and the field measurement of wind field on land and sea is also a research hotspot in the past decade. However, it has great limitations because of its high cost and time consuming, and it cannot be implemented before the construction of structures.

In the physical wind tunnel test, the characteristic value of the fluctuating wind and the characteristic value of the dynamic response on the surface of the structure can be obtained by establishing the structure model. However, the main problem is that it is difficult to make the structure model according to the similarity ratio criterion. Many researchers have obtained parameters for wind load calculation through wind tunnel tests of various structures. Pike and Vickery [6] conducted wind tunnel experiments on a cable tower platform and compared the results with the standard calculation. Troesch [7] et al. carried out wind tunnel tests on the model of a semi-submersible platform to calculated the wind load. Furmes [8] et al. calculated the wind load of ocean oil drilling platform under different wind direction angles and the result was consistent with its wind tunnel test. Pan [9] et al. analyzed the calculation method in classification society and written a program of wind load and wind tilting moment calculation. Lin presents a finite element method for calculating the wind area of platforms. Presently, numerical simulation and wind tunnel test are the main methods to modify and improve the calculation model.

The calculation method of wind load according to classification societies norms in many countries does not take the influence factors into account such as masking effect, wind direction angle, and lift, so that calculation results tend to be conservative, sometimes even much larger than experimental results. It has 
become a problem worth studying how to accurately calculate the wind load of the platform structure in the absence of wind tunnel test condition.

Similar calculation methods are used in the American Bureau of Shipping (ABS) and Norske Veritas (DNV) specifications. However, the actual wind pressure is often much smaller than the design value. Researches have shown that wind loads calculated by the ABS and the DNV can be large than 50\% to the measured at certain wind angles.

The numerical simulation method is faster and cheaper and has a broad prospect in wind vibration analysis of structures. However, it relies on the accuracy of mathematical models, and there are many problems unsolved such as an unsteady aerodynamic force of complex structures, etc.

The numerical simulation method is faster and cheaper and has a broad prospect in wind vibration analysis of structures. However, it relies on the accuracy of mathematical models, and there are many problems unsolved such as an unsteady aerodynamic force of complex structures, etc. Okamura [10] theoretically studied and calculated the wind-induced response of transmission towers. $\mathrm{Gu}$ [11] analyzed the wind-induced response of the circular linear structure under various wind direction angles and wind speeds, taking into account the downwind and cross-wind pulse dynamics and the self-excited forces caused by the structure. Liang and Lou [12] studied the wind vibration and three-dimensional dynamic wind load of the high-rise lattice tower through numerical simulation and wind tunnel tests respectively.

\section{Some Concepts in Wind Load Calculation}

In the Chinese current design specification of the marine platform [13], the equivalent static calculation method and the coefficient value method based on reliability theory are comprehensively adopted, only the across-wind load is calculated while the wind heeling moment is assumed as a cosine function of the heeling angle. With the neglected lift, the marine engineers focus on the determination of wind leaning loads on ocean platforms.

The wind load calculation formula given by the current specification is as follows [13]:

$$
F_{w}=0.613 C_{h} C_{s} S V_{w}^{2}
$$

where, $C_{h}$ is the height coefficient for platform components exposed to wind, which is determined by the vertical distance from the center of the component to the design water surface; $C_{s}$ is the shape coefficient of the component, which is determined by the value in the specification; $S$ is the projected area of the windward components with the platform floating or inclined; $V_{W}$ is the design wind speed.

In the Chinese current design specification of the marine platform [13], the correlation between wind force and wind speed is correct and consistent with the test results while the difference in specific values is large. The calculation method of drag coefficients based on aerodynamics and civil engineering is bas- 
ically reliable. Therefore, the main factors leading to the large difference are omitting the masking effect between components in the shape coefficient calculation, the role of lift, and the underwater resistance center.

The concepts of shading effect, lift effect, shape factor, and center of underwater resistance are briefly introduced in the following sections, as well as the calculation methods for these elements in the specification, to demonstrate the size and number of mistakes in current calculation methods.

\subsection{Shading Effect}

The masking effect is the mutual shielding phenomenon caused by the closer distance between the components when subjected to wind load so that the actual wind of components is less than the total of the individual effect. When determining the projected area of the components in the wind direction, the masking effects between them must be taken into account, especially for semisubmersible platforms, the relationship between the masking effect and shape coefficient of columns is important [14]. However, the current specification does not mention the masking effect between the wind components, such as the shielding of the superstructure at the top of the platform covers the bottom of the derrick when the vertical wind is blowing. Besides, the wind areas are determined by experience instead of formulas, which brings a ring to consider errors to the calculation results [15].

\subsection{Shape Coefficient}

Although there are some differences in shape coefficients stipulated by classification society standards in different countries, the calculation of platforms in the position of positive floating or small inclinations is generally accurate, but the current specifications do not take into account the impact of wind angle of attack. If only the projected area of the wind component is considered to change with the wind, regardless of the change in the shape coefficient, the wind load calculation results will be too conservative [16].

\subsection{Lift}

The large flat surfaces on ocean platforms will generate lift when exposed to wind, such as sitting bottom, self-elevating, and semi-submersible platform deck bottom, etc. When the wind speed is high, the lift will reduce the torque, especially when the tilt angle of the platform is large. Therefore, the results are insufficient and too safe when lift is not considered in the calculation.

\subsection{Underwater Resistance Center}

It is difficult to accurately calculate the underwater resistance center of the platform now. When calculating the vertical distance of the underwater lateral resistance center of a drilling ship, one-half of the edible water can be taken according to the specification. To other types, it is assumed that the center is also the 
center of the shadow area of the underwater part of the platform in the opposite direction of the wind. But this assumption is not reasonable in calculating when the leg of the jack-up platform drops. Besides, the calculation distance from the center of underwater resistance to the surface is related to the wind direction.

It can be seen that the current wind load calculation method of semi-submersible offshore platform is not enough to meet the requirements of future deep-sea offshore platform, especially the wind load calculation mistakes of the upper tower structures. The main reason is that the existing specifications ignore the lift force of the wind on the platform. Therefore, it is necessary to study and analyze the calculation method of lift force of ocean horizontal typhoon.

\section{Lift Calculation Method}

Lift is the ability of the wind to exert an upward force on a plane with an appropriate inclination. The current method for calculating the lift based on fluid mechanics and structural dynamics, according to the different assumptions, calculation method and calculation model generally include the following: based on the random vibration theory of vortex-induced lift model, based on the fluidstructure interaction of structural dynamics calculation method, according to the theory of gas flow pressure calculation method, and numerical simulation method, etc.

\subsection{Lift Correlation Model Established by Blevins and Burton}

Blevins and Burton established the analytical model for calculating vortex induced lift and structural vibration according to the random vibration theory [17]. Assuming it exists that an elastic cylinder moving in a swirl field, according to that the external force work is equal to the lift and the cylindrical deflection, it can be obtained that:

$$
F_{e}(t)=\frac{1}{2} \rho v^{2} D \int_{0}^{L} C_{L}(z, t) \varphi(z) \mathrm{d} z
$$

Define an equivalent lift time function:

$$
C_{L E}(t)=\frac{\int_{0}^{L} C_{L}(z, t) \varphi(z) \mathrm{d} z}{\int_{0}^{L} \varphi(z) \mathrm{d} z}
$$

The equivalent lift is:

$$
F_{e}(t)=\frac{1}{2} \rho v^{2} D C_{L E}(t) \int_{0}^{L} \varphi(z) \mathrm{d} z
$$

If vortex discharge efficiency $w_{s}=2 \pi S t v / D$, the lift coefficient changes over time can be considered harmonic, which is $C_{L}(z, t)=C_{L 0}(z) \sin w_{s} t$, where $C_{L 0 E}=\frac{\int_{0}^{L} C_{L 0}(z) \varphi(z) d z}{\int_{0}^{L} \varphi(z) d z}$.

Considering the most unfavorable state that the resonance caused by the same vortex induced by wind lift and structural frequency, it can be conducted that 
the vibration response spectrum equal to vortex-induced force spectrum multiplied by structural coefficient. If the structural displacement function is expressed as a spectral density function, the expression of the equivalent lift coefficient $C_{L O E}$ is finally obtained:

$$
C_{L O E}=\frac{K_{1}^{2} K_{2}^{2} \int_{0}^{L} \int_{0}^{L} g(z) g\left(z^{\prime}\right) \gamma\left(z-z^{\prime}\right) \varphi(z) \varphi\left(z^{\prime}\right) \mathrm{d} z \mathrm{~d} z^{\prime}}{\left(\int_{0}^{L} \varphi^{2}(z) \mathrm{d} z\right)^{2}}
$$

Finally, all results which considered the non-uniformity of the axial distribution of the structure can be expressed with the lift correlation function $\gamma\left(z-z^{\prime}\right)$ and different modal functions $\varphi(z)$ and the lift can be calculated.

\subsection{Calculation Method Proposed by Engineering Structural Dynamics}

The structure of the ocean platform is complex, and the lift comes from the specific flow field formed by the wind around the structure, which eventually manifests as a strong structural vibration characterized by fluid-solid coupling. The reason may be the wake and vortex of the wind passing through the tail end of the structure, vortex-induced vibration, or by a combination of a particular structure shape and airflow direction which is called jump vibration [18] (also known as overriding vibration, galloping, or flutter). The upper structure of the platform is affected by wind load as the main source of lift, and the position between them should be noted. Therefore, when calculating the wind load, the upper building can be divided into four types of components, and it is split into the rod and plate system.

Drilling towers and lifting equipment are mainly composed of smaller diameter column components, a flow state of the wind can be described by Reynolds number, through the Morison form of formula to calculate the wind load. The house is a closed box, and the wind load on the windward vertical plane is calculated according to the gust factor of Davenport. A structure with a larger horizontal surface, such as a platform deck, may cause flutter at the leading edge of the horizontal surface. The wind load on the horizontal top is mainly the vertical lift. To large columns on the platform, assumptions about cross-correlation function caused by the unequal profile shape of columns and the uneven distribution of wind speed can be made by spectral analysis.

For the rod system structure, the wind lift calculation formula is:

$$
F_{L}=0.5 \rho_{a} C_{L} \bar{v}|\bar{v}|
$$

where, $C_{L}$ is lift coefficient which is also a function of time and depends on the Straha frequency, $f_{s}=S t \cdot v / D$

For the plate structure, $\chi$ which is the aerodynamic admittance coefficient related to turbulent frequency can be inducted, and the wind pressure spectrum can be expressed as the correlation function of wind speed:

$S_{p p}(f)=\chi^{2}\left(2 \frac{p}{u}\right) S_{v v}(f)$. By calculating the pressure cross power spectral den- 
sity function between two points of the structure, it is obtained that:

$$
S_{p p}^{\prime}(f)=\iint_{A} \iint_{A} S_{p p}^{\prime}\left(y_{1}, y_{2}, z_{1}, z_{2}, f\right) \phi_{r}\left(y_{1}, z_{1}\right) \phi_{r}\left(y_{2}, z_{2}\right) \mathrm{d} y_{1} \mathrm{~d} y_{2} \mathrm{~d} z_{1} \mathrm{~d} z_{2}
$$

where, $\phi_{r}(y, z)$ belongs to the $r$ mode.

\subsection{Calculation Method Proposed by Gas Flow Pressure Theory}

According to the air flow pressure formula, the lift comes from the velocity difference between the upper and lower plane of the platform component. Therefore, the lift calculation formula for the vertical component of the platform is proposed:

$$
F_{L}=C_{L} \cdot \frac{1}{2} \frac{\gamma}{g} V^{2} \cdot A_{p}
$$

where, $C_{L}$ is Lift coefficient, $C_{L}$ is related to the section shape of the component, can be obtained by looking up the table. For flexible structures such as cables on the platform, $C_{L}$ is correlative to angle $\theta$ between the cable string and wind direction. In the design manual of U.S. Department of Navy Engineering, the calculation formula of cable lift is as follows:

$$
F_{L}=2.133\left(C_{p} \cdot d \cdot v^{2}\right) \cdot C_{L} \cdot 10^{-3} \mathrm{~s}
$$

where, $C$ is the cable chord length, $d$ is the cable diameter, $v$ is the wind speed at middle height of cable. $C_{L}$ is correlative to angle, and can be achieved by angle $\theta$ in relation curves.

\subsection{Numerical Simulation Method}

In recent years, with the development of numerical simulation and computer operation ability, many researchers and designers calculate the wind load through finite element software when designing offshore platforms [19]. The lift effect of wind on the platform in different environments and the influence of lift on the anti-tilt distance of the platform are obtained.

The fluid mechanics' method (CFD method) solves the wind load on the surface by simulating the change of wind field around the structure by computer. The key point is the calculation of fluid dynamics and numerical simulation. The specific solving process is as follows: a set of differential equations is used to describe the flow state of the fluid in space, discrete the computing region firstly, and convert the differential equations into algebraic equations, and then find the numerical solution of the computational equation system. The wind of the ocean platform can be simulated by the bluff body aerodynamics under turbulence in the atmospheric boundary layer. Turbulence numerical simulation methods include direct numerical simulation and non-direct numerical simulation. The non-direct numerical simulation consists of the large eddy simulation method (LES), Reynolds average method (RANS), and the statistical average method. Among them, Reynolds average method includes the Reynolds stress model and eddy viscosity model.

The basic equations of fluid mechanics include the following three major equ- 
ations: Continuous equation:

$$
\sum_{i=1}^{3} \frac{\partial u_{i}}{\partial x_{i}}=0
$$

Momentum equation:

$$
\rho \frac{D u_{i}}{D t}=\rho F_{i}+\sum_{j=1}^{3} \frac{\partial \sigma_{i, j}}{\partial x_{j}} \quad(i=1,2,3)
$$

Navier-stokes equation:

$$
\rho \frac{D u_{i}}{D t}=-\frac{\partial p}{\partial x_{i}}+\mu \frac{\partial^{2} u_{i}}{\partial x_{j} \partial x_{j}}+\rho F_{i}
$$

Nowadays, designers mainly use Fluent software to calculate the wind load of ocean platforms [20]. It can simulate 2-D and 3-D flow analysis [21], steady and unsteady flow, compressible and incompressible calculation, laminar flow and turbulence, etc. Its turbulence models provided include the Spalart-Allmaras model, standard $k-\varepsilon$ model, modified $k-\varepsilon$ model, Reynolds stress model (RSM), and large eddy simulation (LES), etc.

\subsection{Navier-Stokes Equation Solving Method}

Navier-stokes equation solution methods include direct numerical simulation, time-averaged N-S equations, and model theory, large eddy simulation, etc. The most common engineering method is the "turbulence model" method with some assumptions. The solving process is represented high-order unknown correlation with low order interconnected terms or time-averaged characteristics in the Reynolds time-averaged equation or the transport equation of the turbulent, and the closed Reynolds time-averaged equation is obtained.

Turbulent flow models are divided into three categories. The first two averages the turbulent eddies statistically. The first category is the turbulence transport coefficient model proposed by Boussinesq for 2-D flow. According to the number of established differential equations, it can be divided into a zero equation model (Algebraic equation model), a single equation model, and a two-equation model. The main task is to provide an expression of the turbulence viscosity coefficient $\mu_{i}$ or a method calculating the transport equation. The second type is to discard the turbulence transport coefficient and establish the transport equation of turbulence stress and other second-order correlations directly. The third type is a large eddy simulation, which divides the eddies into large-scale turbulence and small-scale turbulence. By solving the modified Navier-stokes equation, the motion characteristics of the large eddies can be obtained, and the small eddies will be calculated on average by statistical method.

\section{Conclusions}

The existing specification does not calculate the wind-to-ocean platform lift separately, and the calculated wind load is too large. Although its safety is guaranteed, a large amount of material and structural strength is wasted. With the up- 
date and progress of the calculation method, it is necessary to revise this.

The lift model is based on the analysis of vortex-induced effect and structural vibration. The lift is calculated as a vibration load while considering the influence of position and structural frequency. However, the model itself is also based on the assumption of wind and platform resonance, so that this method is suitable for the existence of stable vortex wake.

In the calculation method of the structural dynamics of marine engineering, the designer thinks that the upper building and platform deck are the main sources of lift. The structural components are split into the rod and the plate systems, and they can be calculated separately. This method must be combined with the wind tunnel test and consider the mistakes caused by the position relationship.

In the theoretical calculation method of airflow pressure, based on the assumption that the lift comes from the flow velocity difference between the upper and lower planes of the platform components, the formula is simple and convenient, which is suitable for estimating the lift and can be incorporated into the specification.

The numerical simulation method has been widely used in the design of ocean platforms and the research on the interaction between structure and environmental load. The accuracy of this method is high, but there is no general calculation formula or program. Therefore, each platform needs to be remodeled and programmed, resulting in tedious design work, which is not universal.

\section{Conflicts of Interest}

The author declares no conflicts of interest regarding the publication of this paper.

\section{References}

[1] Tang, Y. (2008) Marine Structural Dynamics. Tianjin University Press, Tianjin, 14-57. (In Chinese)

[2] Sun, Y. (1989) Environmental Conditions and Loads of Ocean Engineering. Shanghai Jiaotong University Press, Shanghai, 16-48. (In Chinese)

[3] Pan, B. and Yuan, J. (1997) Wind Tilt Load Acting on the Ocean Mobile Platform. The Ocean Engineering, 15, 32-37.

[4] Li, F., Zhou, D., Ma, J., et al. (2017) Wind Load Simulation and Dynamic Response Analysis on a Semi-Submersible Platform under Environmental Wind and Wave Loads. Journal of Vibration and Shock, 36, 112-117.

[5] Shi, J. (2009) The Study of Wind Load on Offshore Platform. Dalian University of Technology, 5, 9-11.

[6] Pike, P.J. and Vickery, B.J. (1982) A Wind-Tunnel Investigation of Loads and Pressures on a Typical Guyed Tower Offshore Platform. Offshore Technology Conference, Houston, May 1982, Paper No. OTC-4288. https://doi.org/10.4043/4288-MS

[7] Troesch, A.W., Vangunst, R.W. and Lee, S.W. (1983) Wind Loads on a 1:115 Model of a Semisubmersible. Maritime Technology, 20, 283-289.

https://doi.org/10.5957/mt1.1983.20.3.283 
[8] Furnes, G.K. (1998) Numerical Simulations of Wind Forces on Troll B. Marine Structures, 11, 273-289. https://doi.org/10.1016/S0951-8339(98)00013-6

[9] Mu, J. and Pan, B. (1999) Analysis and Calculation of Wind Load on Offshore Platform. China Offshore Platform, 14, 7-11, 46.

[10] Wang, Q. and Gu, M. (2010) Analysis Method of Wind Induced Response of Circular Linear Structures. Journal of Vibration Engineering, 23, 151-157.

[11] Okamura, T., Ohk uma, T., Hongo, E. and Okada, H. (2003) Wind Response Analysis of Transmission Tower in a Mountainous Area. Journal of Wind Engineering and Industrial Aerodynamics, 91, 53-63. https://doi.org/10.1016/S0167-6105(02)00322-7

[12] Liang, S., Zou, L. and Zhao, L. (2007) Wind Tunnel Test Study on Three Dimensional Dynamic Wind Load of Lattice Tower. Journal of Aerodynamics, 25, 311-318.

[13] China National Standardization Management Committee (2018) Petroleum and Natural Gas Industries Floating Offshore Structure. China Standardization Press, Beijing, 19-21.

[14] Cao, J. (2015) Research on Overall Design of Liftboat Platform. Transportation Science \& Technology, 270, 182-184.

[15] Ma, H., Liu, S., Gu, Y., et al. (2004) Layout Optimization of Offshore Platform Structures Considering Design Rules. Chinese Journal of Computational Mechanics, 1, 38-44, 87.

[16] Wang, Y. and Wang, M. (2018) Study on Wind Loads on Offshore Platforms with Consideration of Shielding Effect. Ship Engineering, 40, 298-302.

[17] Jiang, R. (2016) Numerical Simulation Calculation of Wind Load on Semi-Submersible Drilling Service Support Platform. Master's Thesis, Jiangsu University of Science and Technology, Zhenjiang, 84-107.

[18] Pan, Y. (2004) Numerical Simulation of Two-Dimensional Flow Past a Tall Building Using a Discrete Vortex Method. Huazhong University of Science \& Technology, 2, 9-46.

[19] Zhang, X. (2006) Structural Wind Engineering. China Architecture \& Building Press, Beijing, 110-154.

[20] Lin, Y., Hu, A. and Jiang, W. (2014) Numerical Analysis of Wind Load for Offshore Platform. Ship Engineering, 36, 104-108.

[21] Ma, Z. and Wang, X. (2009) Numerical Simulation Calculation of Wind Load on Deep Water Semi-Submersible Platform. Science \& Technology Association Forum, 8, 98-99. 